\title{
Knowledge Management in Practice: A Special Issue
}

\author{
Carla Simone ${ }^{1}$, Mark Ackerman ${ }^{2} \&$ Volker Wulf $^{3}$ \\ ${ }^{1}$ University of Milano Bicocca, Milan, Italy (E-mail: simone@disco.unimib.it); ${ }^{2}$ University of \\ Michigan, Ann Arbor, USA; ${ }^{3}$ University of Siegen, Siegen, Germany
}

Organizations of different kinds, from structured companies up to social networks or virtual communities, are becoming increasingly aware of the need to collect, organize, mobilize, and increase the expertise and knowledge which characterize their ability to stay alive, adapt, and evolve in a turbulent context. Knowledge Management $(\mathrm{KM})$ is the current term for the different organizational and technological approaches to answer this need. KM, from a management perspective, is an attempt to rationalize and manage the vast amounts of formal and informal knowledge that any organization, especially large companies, has. $\mathrm{KM}$, from a practice perspective, investigates the everyday practices that lead to organizationally situated use of that formal and informal knowledge (Ackerman et al. 2008). While the KM discourse has long been focusing on opportunities to externalize and represent knowledge in artifacts, the identification of knowledgeable actors and the support of social networks as well as their interactions with artifact becomes highly relevant in a practice perspective (Ackerman et al. 2003).

$\mathrm{CSCW}$ has examined knowledge and information in organizations from its very beginnings. In more recent years, the $\mathrm{CSCW}$ community has examined $\mathrm{KM}$ per se. This manifested itself in asking for applied research in the KM practices of real organizations instead of blindly following media hype - the critical realism stance that Robert Kling advocated over the technological utopianism of the media and technology promoters. In addition, the KM investigation in CSCW has followed CSCW's notable interest in discussing and detailing the interrelationships between technological and organizational innovations, and $\mathrm{CSCW}$ as a community has taken on the task of considering those interrelationships critically. This views KM largely as a matter of socio-technical design - and a difficult design problem at that.

This special issue adheres to these positions. Our aim was to collect articles reporting on knowledge management in action. We wanted to further the discussion of the issues of KM through the kinds of fine-grained ethnography-based investigations found in this community and this journal. Specifically, we wanted ethnography-based or other interpretivist work that confronted and learned from real organizational situations. We wanted these papers to highlight the problems, requirements, tradeoffs, and technical solutions in KM that can be derived only from field-based research. 
In this volume, we are fortunate to have six articles that discuss KM issues in the depth and detail that $\mathrm{CSCW}$ requires. Two of them deal with two very popular concepts in KM: community of practice and tacit knowledge; they discuss the origins of these concepts and their influence on the KM field from the business and research standpoints. Two articles take an empirically grounded design perspective. The first article discusses the implication of the separation between technologies that support information and the ordered flow of work and technologies that support knowledge management. The second article proposes analytical and methodological frameworks to guide the design of technologies for expertise management, which are based on the notion of ecosystem to focus on the interaction between two mutually intertwined elements - artifacts and actors. The last two articles report on field studies in three organizations and in three IT teams of a regional hospital. The latter suggests that not only seeking for knowledge should be supported but also the cooperation among the knowledge seekers in order to take into account how KM is affected by subtle features of the organization. The former article discusses the usage of WIKI technologies from both the management and the end-users point of view by comparing the results of the investigation in the three different organizations.

We have shown, through this special issue and our own work, that information processes in general and knowledge management in particular can be understood best through field-based investigations. Our aim is to detail how the technical and the social intertwine. We believe the way forward includes many more field-based and design-oriented studies so as to understand:

- the specialized needs of important categories of organizations and institutions, such as in educational, medical, or safety-critical domains;

- the impact of culture on knowledge-intense activities, including the strategies for knowledge management;

- how organizations use informal mechanisms of knowledge production, adaptation, and reuse and how these practices could be technically supported;

- persistent issues in technology-use for knowledge management, such as classification and ontologies;

- fitting design concepts and architectures and how they are appropriated in specific social contexts;

- the organizational processes that lead to transformative uses of knowledge management technologies and processes;

- the rhythms of knowledge production and use in organizations.

\section{References}

Ackerman, M.; Pipek, V.; Wulf, V. (eds.): Sharing Expertise: Beyond Knowledge Management. Cambridge, Mass.: MIT-Press, 2003.

Ackerman, M; Dieng-Kuntz, R.; Simone, C.; Wulf, V. (eds.): KMIA 2008: Proceedings of the Conference on Knowledge Management in Action, IFIP Series vol. 270. Boston, Mass.: Springer, 2008. 